\title{
BMJ Open Introducing the non-invasive prenatal testing for detection of Down syndrome in China: a cost-effectiveness analysis
}

\author{
Wenru Shang, ${ }^{1,2}$ Yang Wan, ${ }^{3}$ Jianan Chen, ${ }^{4}$ Yanqiu Du, ${ }^{1,2}$ Jiayan Huang (D) ${ }^{1,2}$
}

To cite: Shang W, Wan Y, Chen J, et al. Introducing the non-invasive prenatal testing for detection of Down syndrome in China: a cost-effectiveness analysis. BMJ Open 2021;11:e046582. doi:10.1136/ bmjopen-2020-046582

- Prepublication history and additional supplemental material for this paper are available online. To view these files, please visit the journal online. To view these files, please visit the journal online (http://dx.doi. org/10.1136/bmjopen-2020046582).

Received 06 November 2020 Accepted 23 June 2021

Check for updates

(C) Author(s) (or their employer(s)) 2021. Re-use permitted under CC BY-NC. No commercial re-use. See rights and permissions. Published by BMJ.

${ }^{1}$ School of Public Health, Fudan University, Shanghai, China

${ }^{2}$ Key Lab of Health Technology

Assessment, National Health

Commission of the People's

Republic of China, Shanghai,

China

${ }^{3}$ Department of gynaecology

and obsterics, Fuyang People's

Hospital (North Campus),

Fuyang, Anhui, China

${ }^{4}$ Department of Health

Management and Health

Economics, University of Oslo, Oslo, Norway

Correspondence to

Dr Jiayan Huang;

jiayanhuang@fudan.edu.cn

\section{ABSTRACT}

Objective This study aimed to compare the health economic value of a non-invasive prenatal testing (NIPT) strategy against a second-trimester triple screening (STS) strategy for the detection of Down syndrome based on real-world data from China.

Design A decision-analytical model was developed to compare the cost-effectiveness of five strategies from a societal perspective. Cost and probability input data were obtained from the real-world surveys and published sources.

Setting China.

Participants Women with a singleton pregnancy. Interventions The five strategies for screening were: (A) maternal age with STS (no NIPT); (B) STS plus NIPT screening; (C) age-STS plus NIPT screening (the currently referral strategy in China); (D) maternal age with NIPT screening and (E) universal NIPT screening.

Main outcome measures Incremental cost-effectiveness ratios (ICERs) per additional Down syndrome case terminated, univariate and probabilistic sensitivity analysis and cost-effectiveness acceptability curves were obtained. Results Strategy A detected the least number of Down syndrome cases. Compared with the cheapest Strategy B, Strategy D had the lowest ICER (incremental cost, US\$98 944.85 per additional Down syndrome case detected). Strategy $D$ had the highest probability of being costeffective at the willingness-to-pay level between US $\$ 110$ 000.00 and US\$535 000.00 per additional Down syndrome case averted. Strategy E would not be cost-effective unless the unit cost of the NIPT could be decreased to US\$60.50.

Conclusion Introducing NIPT screening strategies was beneficial over the use of STS strategy alone. Evaluating maternal age in combination with the NIPT screening strategy performs better than China's currently referral strategy in terms of cost-effectiveness and safety. Lowering the price of NIPT and optimising payment methods are effective measures to promote universal NIPT strategies in China.

\section{INTRODUCTION}

Down syndrome, which is caused by trisomy 21 , is the most frequently occurring autosomal aneuploidy and is associated with delayed physical growth, neurocognitive retardation and other medical issues. ${ }^{1}$ Antenatal screening for Down syndrome, prior

\section{Strengths and limitations of this study}

- From a societal perspective, this study compared the costs and health outcomes of five different screening strategies for Down syndrome, which cover the introduction of non-invasive prenatal testing strategies and second-trimester triple screening (STS) strategy in China.

- Using context-specific real-world information on probability and cost parameters from a survey in Fuyang City allowed an accurate evaluation in a policy-specific context.

- The findings may provide evidence for other developing countries where STS is more common than first-trimester combined screening in clinical practice.

- Considering the differences in the clinical practice and cost of Down's screening across countries, evidence of effectiveness must be cautiously interpreted when applied to other settings.

to a definitive diagnosis, is a routine practice in many countries. Traditional screening methods are based on combinations of advanced maternal age and maternal serum biomarkers, with or without ultrasonographic 'nuchal translucency (NT)' during the first and/or second trimester. ${ }^{2}$ Novel non-invasive prenatal testing (NIPT) is a promising technology that uses cell-free fetal DNA that originates from the placenta and is present in the maternal blood between weeks 11 and 22 of pregnancy. NIPT can ensure more significant prenatal detection of Down syndrome (detection rate (DR) of approximately 99\%) and a lower false-positive rate (FPR; $0.0 \%-0.2 \%$ ) than conventional screening tests, such as first-trimester combined screening (FTCS; NT with maternal serum biomarkers; DR of 92\%; FPR of $7 \%$ ) and second-trimester triple screening (STS) (DR of $79 \%$, FPR of $4 \%$ ). ${ }^{3-5}$ These advantages contribute to the mitigation of the risk associated with invasive diagnostic testing. ${ }^{6}$ Increasingly, guidelines in a number of countries support the use of NIPT for prenatal screening in pregnant women. ${ }^{7-9}$ 
Table 1 Five prenatal screening strategies in the model

\begin{tabular}{|c|c|c|}
\hline Strategies & Name of the strategy & Components of prenatal screening strategies for Down syndrome \\
\hline Strategy A & $\begin{array}{l}\text { Maternal age with STS (age-STS) } \\
\text { screening }\end{array}$ & $\begin{array}{l}\text { 1. For women } \geq 35 \text { years of age, the invasive diagnostic tests } \\
\text { (amniocentesis (AC) or percutaneous umbilical cord puncture for the } \\
\text { prenatal diagnosis) were directly offered. } \\
\text { 2. For women }<35 \text { years of age, STS (nuchal translucency (NT), } \\
\text { maternal serum markers such as unconjugated estriol (UE3), free } \\
\beta \text {-human chorionic gonadotrophin ( } \beta \text {-HCG) and alpha-fetoprotein } \\
\text { ( } \alpha \text {-FP) in the second trimester) was offered; and women who were } \\
\text { determined to have intermediate-risk or high-risk group by STS } \\
\text { (a cut-off between } 1 / 1000-1 / 270 \text { and } \geq 1 / 270 \text {, respectively) would } \\
\text { undergo the invasive diagnostic tests (figure } 1 \text { ). }\end{array}$ \\
\hline
\end{tabular}

Strategy B $\quad$ STS plus NIPT screening

All pregnant women in 14-22 gestational weeks were offered the STS test. Women with screening results indicating an intermediate-risk or high-risk of a fetus with Down syndrome were considered for further NIPT screening; only women testing NIPT-positive would undergo the invasive diagnostic tests (online supplemental appendix figure a).

\begin{tabular}{|c|c|c|}
\hline Strategy C & Age-STS plus NIPT screening & $\begin{array}{l}\text { 1. All women } \geq 35 \text { years of age were directly offered the invasive } \\
\text { diagnostic tests. } \\
\text { 2. Women }<35 \text { years of age were offered the STS test, and } \\
\text { intermediate-risk or high-risk pregnancies were considered for } \\
\text { further NIPT screening; only women testing NIPT-positive would } \\
\text { undergo the invasive diagnostic tests (online supplemental appendix } \\
\text { figure b). }\end{array}$ \\
\hline
\end{tabular}

NIPT, non-invasive prenatal testing; STS, second-trimester triple screening.

Compared with FTCS, STS is the most widely performed screening method in mainland China because of the availability and convenience of this test. ${ }^{5}$ In clinical practice, most pregnant women can easily miss undergoing primary screening in the first trimester. Moreover, the NT test, which requires experts with demonstrated expertise in fetal ultrasound, is not widely available in the settings with scarce health resources and a large population. Therefore, screening in the second trimester is commoner than that in the first trimester. In recent years, NIPT with higher safety and accuracy has become available in China. The National Health Commission (formerly China's National Health and Family Planning Committee) has, since 2016, specified guidelines for the use of high-throughput, NIPT for autosomal aneuploidy. ${ }^{10}{ }^{11}$ Currently, in China, for pregnant women in the second trimester, NIPT is offered as an optional test for those women who (1) are younger than 35 years, and (2) have STS results indicative of intermediate or high risk; however, an invasive test is offered for pregnant women aged 35 years or older directly. ${ }^{12}$
There is a trend to include NIPT under public medical insurance coverage; however, currently NIPT is not covered by insurance or is only partially reimbursed in most countries. ${ }^{1314}$ Moreover, the cost of NIPT varies widely, which creates a potential financial burden for pregnant women in low-income and middle-income countries. ${ }^{15}$ These factors may further limit the widespread use of NIPT. ${ }^{16}$ In China, the price of NIPT in the private sectors varies from US\$202.49 to US\$332.46, as the cost is only partially covered by health insurance in most provinces, pregnant women may have to bear the cost for this service. However, STS costs less than US\$45.33, is covered by health insurance or local government policies in most provinces, and pregnant women are more likely to choose STS over NIPT, despite the higher DR of NIPT for Down syndrome screening. ${ }^{4517}$ Therefore, the clinical application of NIPT has been partly restricted. In order to maximise the advantages of NIPT and improve its clinical application, in April 2017, the government initiated a free NIPT service programme in Fuyang City, located in 


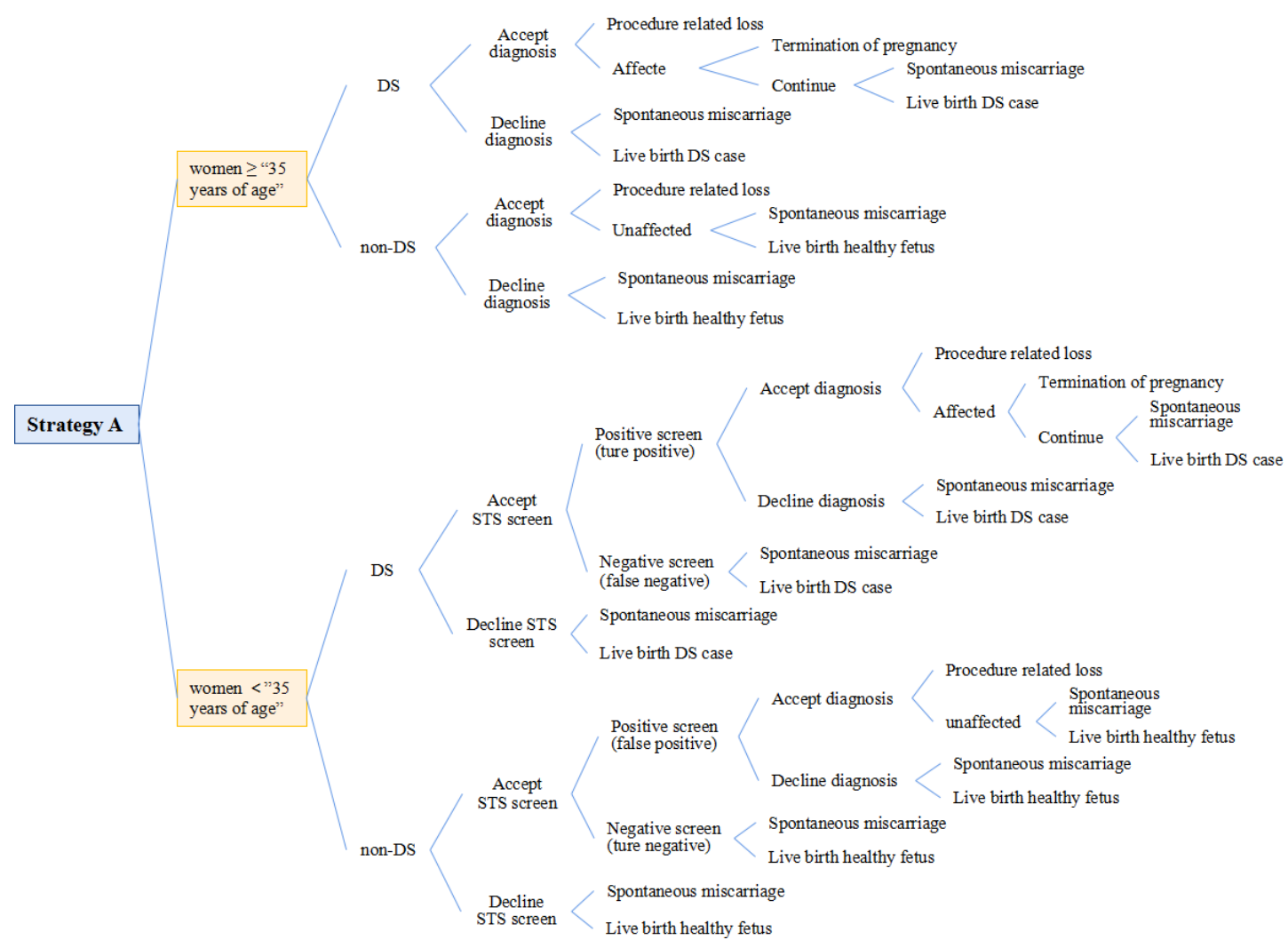

Figure 1 An algorithm of the flow of pregnant women for screening strategies. Strategy A, testing flow of pregnant women when combining maternal age with the STS (age-STS) screening strategy. DS, Down syndrome; STS, second-trimester triple screening.

eastern China, whereby through negotiation with private sector, the local government purchased NIPT services for US $\$ 112$ per case and provided free fetal chromosome aneuploidy (trisomy 21, 18 and 13) screening for all pregnant women. The potential cost and performance of implementing Fuyang's free NIPT service programme for first-line screening of Down syndrome remains to be evaluated. Therefore, healthcare authorities have been concerned whether this screening strategy can be widely implemented as compared with the referral strategy.

The cost-effectiveness among the available prenatal screening strategies for Down syndrome is unapparent, given the diversity across different health service systems and analytical perspectives. Walker $e t a l^{18}$ indicated that the use of NIPT for first-line testing is beneficial in terms of the number of Down syndrome cases detected and the number of miscarriages, despite the considerably higher cost than the first-trimester and second-trimester screening tests. ${ }^{18}$ A recent systematic review stated that universal NIPT is more effective and costlier than the usual screening, and that the cost-effectiveness of contingent NIPT is uncertain, given that NIPT ranges from being dominant to a dominated strategy. ${ }^{19}$ Furthermore, the cost-effectiveness results are significantly affected by analytical perspectives, test accuracy, uptake rate of the tests and the unit cost of NIPT in the sensitivity analysis. ${ }^{19}{ }^{20}$ Most studies adopted FTCS as a comparator, despite its good performance, is not widely implemented in some developing countries because of resource constraints. Moreover, previous studies only considered direct medical costs during the screening procedure. Thus, the previously reported results do not provide enough evidence for developing countries.

This study aimed to address these gaps in clinical practice and the existing cost-effectiveness studies with the following objectives: (1) to compare the costs and performance outcomes of prenatal screening with STS and NIPT within a singleton pregnancy population from a societal perspective; (2) to identify whether China's referral strategy is optimal and (3) to determine whether universal NIPT screening can be promoted based on government-funded projects.

\section{METHODS \\ Decision analytic model}

The decision-analytical model was constructed using decision analysis software (TreeAge Pro 2019). The costs and health outcomes were assessed using five prenatal screening strategies for Down syndrome from a societal perspective.

The five strategies compared in the analysis were: (1) Strategy A: maternal age with STS (age-STS) screening (only STS); (2) Strategy B: STS plus NIPT screening (NIPT as a second-line test); (3) Strategy C: age-STS plus 
NIPT screening which is recommended by the China National Health Committee; (4) Strategy D: maternal age with NIPT screening and (5) Strategy E: the universal NIPT strategy, which is fully funded by the local government and is currently implemented in Fuyang City. The pregnant women pathways for all strategies and the model structure for Strategy A are described in table 1 and figure 1, and the details of the model structure are reported in online supplemental appendix 1 in the supplementary materials. In all strategies, ultrasonographic NT measurements were excluded from the model because they would not be widely available in China. Our study focused only on fetal trisomy 21, although NIPT has higher sensitivity and specificity in screening for trisomy 18 and 13 than the traditional screening tests. ${ }^{21}$ Trisomy 21 is the most common birth defect that is being screened for and supported by clinical standards. Furthermore, the fetal survival rates in trisomy 13 and 18 are very low (frequently, they would result in spontaneous abortion), therefore, the benefits of NIPT are relatively limited as compared with those for trisomy 21.

Six health outcomes were calculated: (1) Down syndrome cases that were detected and terminated with the informed consent of the pregnant women; (2) livebirth Down syndrome cases; (3) spontaneous miscarriage of a Down syndrome fetus; (4) live birth of a healthy fetus; (5) spontaneous miscarriage of a non-Down syndrome fetus; (6) procedure-related losses (PRLs) caused by invasive diagnostic test (figure 2).

\section{Data sources}

Table 2 lists the relative parameters included in the model. The population-based data and the uptake rate of prenatal screening and diagnosis tests for Down syndrome were calculated based on official records from April 2017 to October 2018 that were provided by Fuyang City People's Hospital (FCPH), Fuyang Health Commission and Fuyang Maternal and Child Healthcare Hospital. In 2018, the total number of pregnant women was approximately 100000 , of which $6.85 \%$ were associated with advanced maternal age ( $\geq 35$ years of age). The calculation showed that $80 \%$ of women with a singleton pregnancy (including women $\geq 35$ years of age) underwent NIPT. According to previous studies, the sensitivity and specificity of NIPT for trisomy 21 were $99.50 \%$ (95\% CI $98.7 \%$ to $99.8 \%$ ) and $100 \%$ (95\% CI $99.9 \%$ to $100 \%$ ), respectively. ${ }^{4}$ For STS, the prenatal screening uptake rate was assumed to be $75 \%$, and the sensitivity and specificity were $79 \%$ (95\% CI $72 \%$ to $85 \%$ ) and $96 \%$ (95\% CI 94\% to 97\%), respectively. ${ }^{5}$ Moreover, $60 \%$ of the pregnant women with a high probability of aneuploidy on STS were assumed to opt directly for invasive diagnostic tests. Meanwhile, it is assumed that none of the women with a high probability of aneuploidy after NIPT declined invasive diagnostic testing. Overall, $95 \%$ of women underwent elective abortion due to a diagnosis of trisomy 21 that was confirmed by invasive diagnostic tests. With regard to the adverse effects associated with invasive diagnostic tests, the proportion of PRLs was $0.30 \% .{ }^{6}$

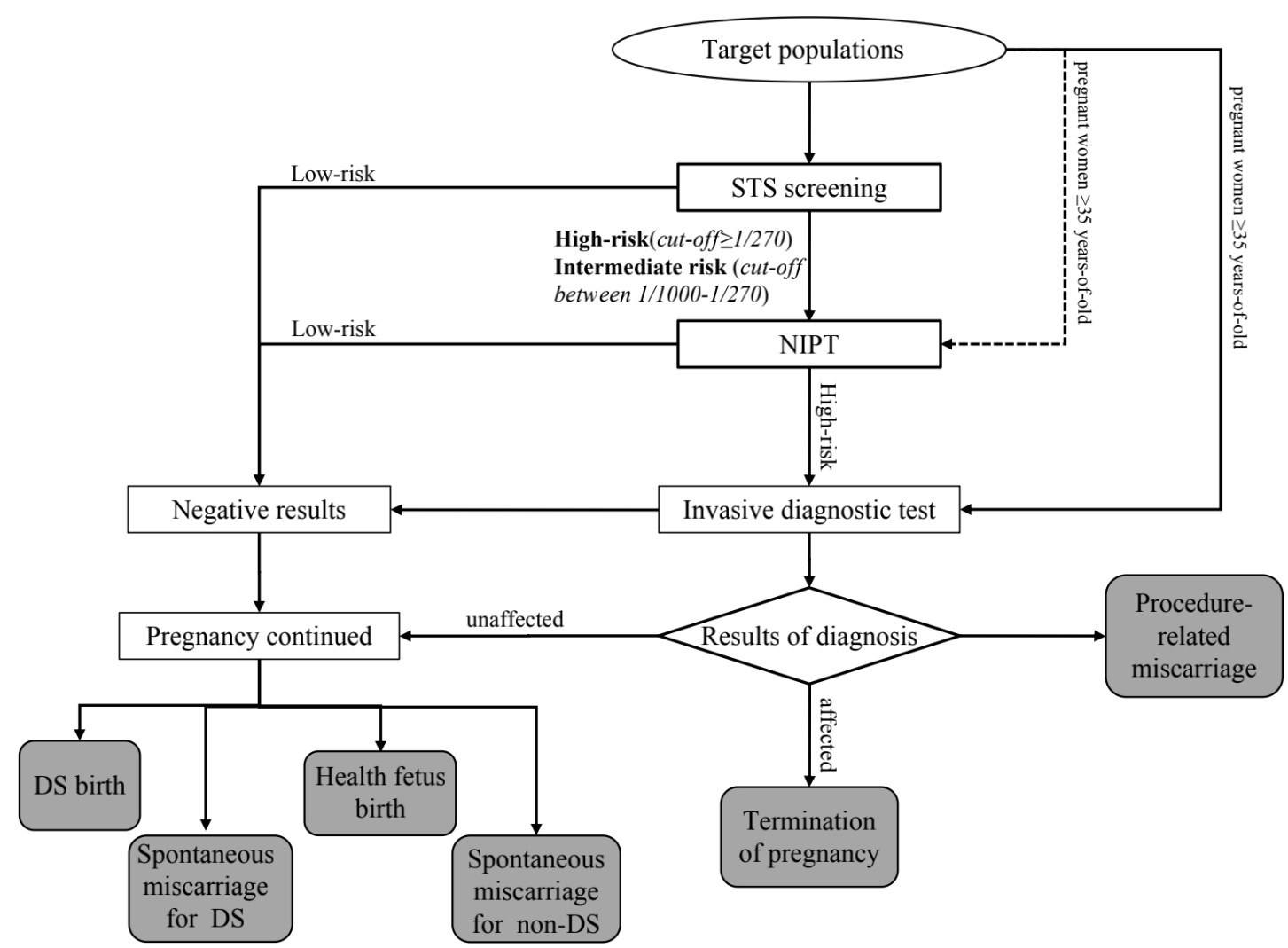

Figure 2 Six health outcomes included in the prenatal screening pathway for Down syndrome fetuses. DS, Down syndrome; NIPT, non-invasive prenatal testing; STS, second-trimester triple screening. 
Table 2 Relative variables in the decision-analytical model

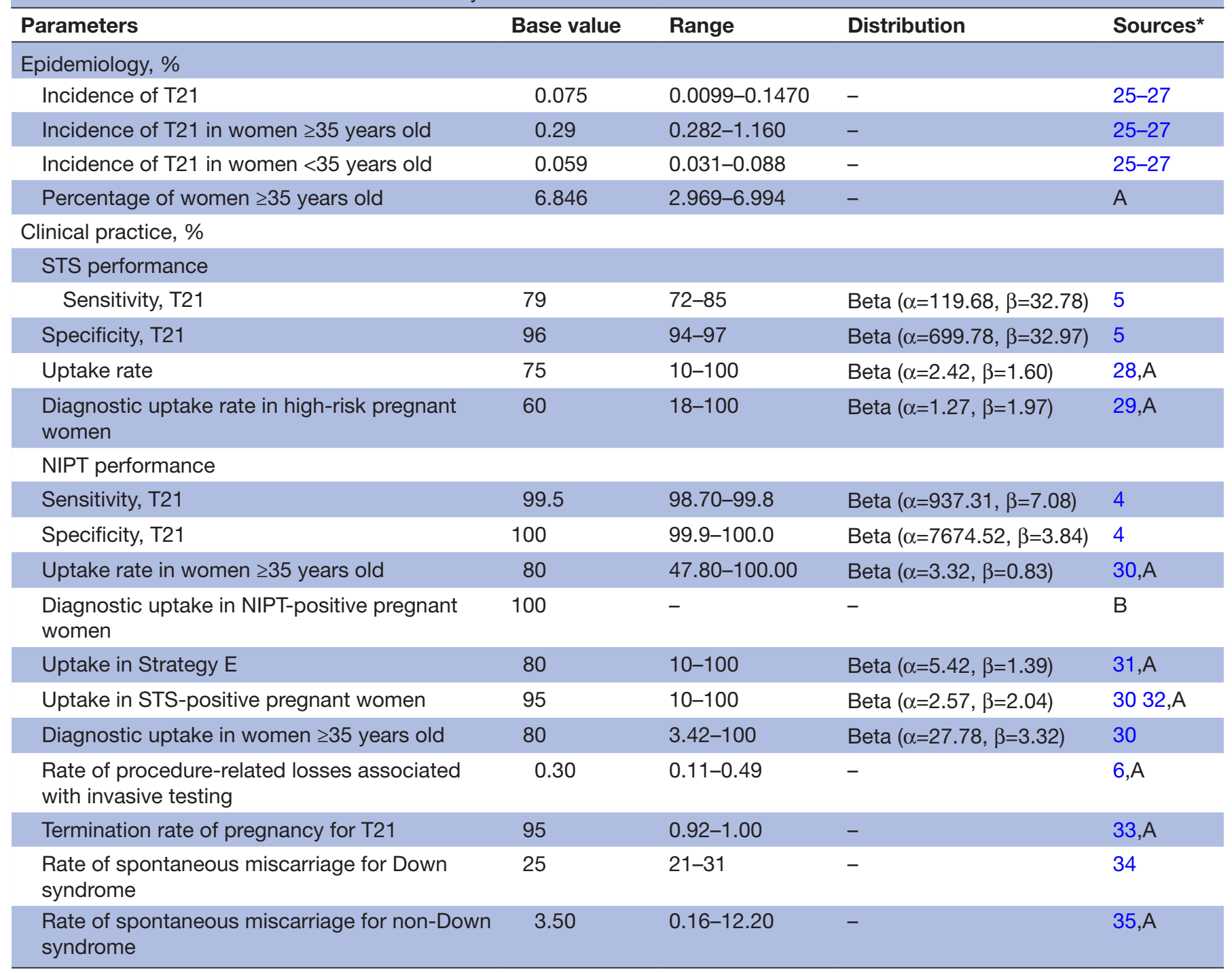

*Data sources: A means data from local official statistics; B means model assumption based on expert opinion. NIPT, non-invasive prenatal testing; STS, second-trimester triple screening; T21, trisomy 21.

In the model, both direct medical costs and direct nonmedical and indirect costs were considered from a societal perspective (table 3 ). The direct medical costs of health service items were replaced by the local list prices of the FCPH. Among them, the NIPT service was purchased by the local government at US $\$ 112.58$ per case. The direct non-medical costs and indirect costs were obtained from a questionnaire survey of 1400 maternal women in FCPH form March to June 2019, which included lost earnings for pregnant women and caregivers due to missing work, transportation costs and accommodation costs during the process of prenatal screening, diagnosis and childbirth. The EpiData database was used to manage the questionnaire survey data, and the mean cost was calculated using MS Excel. The cost parameters are presented in table 3 . The model adopted a short-term (1 year) time horizon, therefore, long-term effects related to infants with Down syndrome were not considered, and the costs were not discounted. All costs were calculated in Chinese Yuan but converted and presented in US\$ (using the 2018 yearly average currency exchange rate: $6.6174 \mathrm{CNY}=1 \mathrm{US} \$$ ).

\section{Economic analysis}

A cohort of 100000 pregnant women was simulated in the model, corresponding to the current estimated annual number of pregnant women in Fuyang City. The effectiveness and total cost of each screening strategy were calculated. Effectiveness was measured in terms of the number of Down syndrome cases that ended in the termination of pregnancy with the informed consent of the pregnant woman. The cost-effectiveness (CE) ratio was defined as the cost per case of Down syndrome that was averted. Meanwhile, to determine which strategy was optimal, the incremental cost-effectiveness ratios (ICERs) were calculated between each strategy and the cheapest strategy. The ICER refers to the difference in the costs 
Table 3 Relative variables in the decision-analytic model

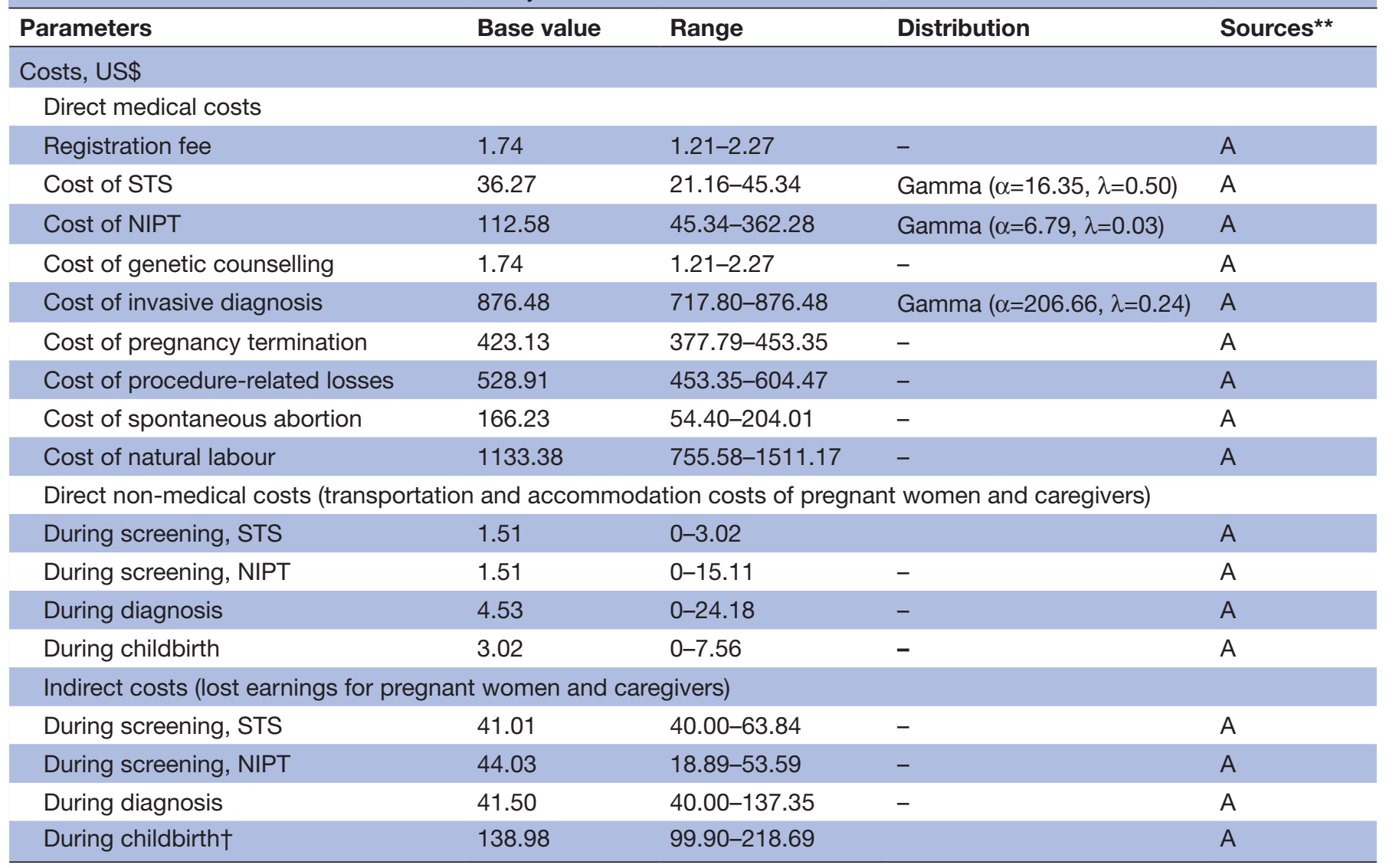

${ }^{*}$ Data sources: A means data from local official statistics.

†Calculation of the indirect costs of lost earning during childbirth only includes caregivers, because lost earning for pregnant women in China is covered by maternity insurance.

NIPT, non-invasive prenatal testing; STS, second-trimester triple screening.

between the two strategies divided by the difference in the number of Down syndrome cases detected by the two strategies $(\mathrm{ICER}=\Delta \mathrm{C} / \Delta \mathrm{E})$. This ratio indicates the incremental cost of using one screening strategy compared with the other per additional case of Down syndrome that is averted.

\section{Sensitivity analyses}

Univariate sensitivity analyses and probabilistic sensitivity analyses (PSA) were conducted. One-way sensitivity analyses were conducted to determine the impact of relevant parametric values on the cost-effectiveness ratios. The parameters were determined according to the available literature evidence and expert opinions. The impacts of the following changes on the outcomes were assessed: first, NIPT when used as a part of the prenatal screening, its uptake rate could increase as a result of exemption of out-of-pocket expenses; second, the cost per NIPT could range from US $\$ 45.34$ to US $\$ 362.28$. PSA were conducted to determine the overall uncertainty by repeating the Monte Carlo simulation 1000 times. For each repetition, new parametric values were obtained from the parameter distributions shown in tables 2 and 3. Gamma distributions were fitted to costs, whereas probability parameters were drawn from the beta distributions. The cost-effectiveness acceptability curves (CEACs) are presented graphically.

\section{Patient and public involvement}

The pregnant women were not invited to comment on the study design and were not consulted to interpret the results. The pregnant women were not invited to contribute to the writing or editing of this paper.

\section{RESULTS}

\section{Economic analysis}

The results for each of the Down syndrome screening strategies are listed in table 4. Strategy A resulted in the detection of 33.55 Down syndrome cases, with a greater number of live-birth Down syndrome cases (30.87), and the greatest number of PRL cases of 21.52. The number of Down syndrome cases detected for Strategy B was 39.78 , and the number of PRL cases was only 0.13. Moreover, Strategy B was the cheapest strategy, with a total cost of US $\$ 7671818.41$. Strategies C and D resulted in 44.20 and 44.12 Down syndrome cases detected, respectively. Nevertheless, Strategy C resulted in more PRL cases than Strategy D (16.52 vs 0.14). Strategy E resulted in 
Table 4 Cost-effectiveness analysis results

\begin{tabular}{|c|c|c|c|c|c|}
\hline Screening and diagnostic strategy & Strategy A & Strategy B & Strategy C & Strategy D & Strategy E \\
\hline $\begin{array}{l}\text { Number of spontaneous miscarriages } \\
\text { for Down syndrome cases }\end{array}$ & 10.29 & 8.77 & 7.62 & 7.64 & 4.57 \\
\hline $\begin{array}{l}\text { Number of procedure-related } \\
\text { pregnancy losses }\end{array}$ & 21.52 & 0.13 & 16.52 & 0.14 & 0.18 \\
\hline Number of live births of healthy fetuses & 96407.14 & 96427.63 & 96455.68 & 96427.80 & 96427.63 \\
\hline Total & 100000.00 & 100000.00 & 100000.00 & 100000.00 & 100000.00 \\
\hline Incremental effectiveness & -6.24 & - & 4.41 & 4.34 & 16.76 \\
\hline ICER & -920135.75 & - & 1044629.76 & 98944.85 & 375487.45 \\
\hline
\end{tabular}

Strategy A, maternal age with STS (age-STS) screening, Strategy B, STS plus NIPT screening, Strategy C, age-STS plus NIPT screening, Strategy D, maternal age with NIPT screening and Strategy E, universal NIPT screening.

ICER, incremental cost-effectiveness ratio; NIPT, non-invasive prenatal testing; STS, second-trimester triple screening.

the detection of the highest number of Down syndrome cases (56.54) and the fewest number of live-birth Down syndrome cases (13.71). The total cost of Strategy E was US $\$ 13964988.03$. table 4 shows the incremental costs and outcomes using Strategy B as the comparator. Strategy D has the lowest ICER, with an incremental cost of US $\$ 98$ 944.85 per additional Down syndrome case detected.

Figure 3 shows that Strategies A and C were absolutedominated and extended-dominated strategy, respectively,

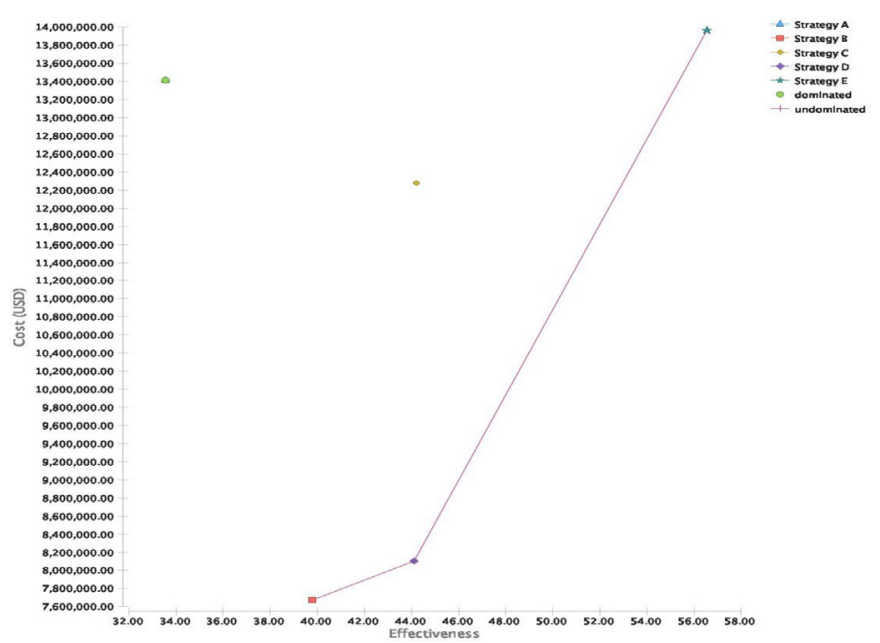

Figure 3 Cost-effectiveness analysis results. Strategy A: maternal age with STS (age-STS) screening, Strategy B: STS plus NIPT screening, Strategy C: age-STS plus NIPT screening, Strategy D: maternal age with NIPT screening and Strategy E: universal NIPT screening. NIPT, non-invasive prenatal testing; STS, second-trimester triple screening. which might be rejected as alternative strategies. In contrast, Strategies B, D and E could possibly to be ideal in different contexts.

\section{Univariate sensitivity analyses}

Univariate sensitivity analysis was performed on the key variables using the ranges shown in tables 2 and 3. In the one-way sensitivity analysis, when the screening uptake rate with STS in pregnant women exceeded $53.00 \%$, the number of Down syndrome cases detected in Strategy B surpassed those in Strategy A, and the number of Down syndrome detected in Strategies D and C was always better than the number of cases detected in Strategy B (figure 4). When the screening uptake rate with NIPT in advanced maternal age pregnancies exceeded $80.00 \%$, the number of Down syndrome cases detected in Strategy D would surpass those of Strategy C (figure 5). When the NIPT uptake rate in STS-positive pregnant women exceeded $60.00 \%$, the number of Down syndrome cases detected in Strategies C and D would surpass those of Strategy A. When this value exceeded $80 \%$, the effectiveness of Strategy B would exceed that of Strategy A. The effectiveness of Strategies C and D was always better than that of Strategy B (figure 6). When NIPT was adopted as a first-line screening strategy for all pregnant women, the effectiveness of Strategy E would overtake Strategies $\mathrm{C}$ and $\mathrm{D}$ if the uptake rate of NIPT exceeded $62.53 \%$ (figure 7). When the price of NIPT was between US $\$ 60.60$ and US $\$ 193.70$, Strategy D had the lowest CE ratio. Moreover, when the cost of NIPT decreased to US $\$ 60.50$ or less, Strategy E showed the lowest CE ratio and surpassed 


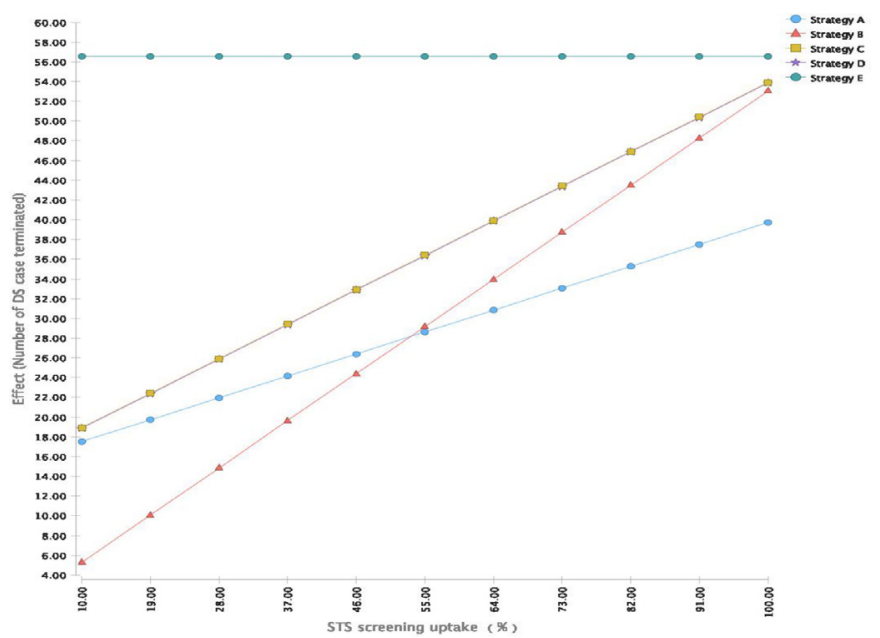

Figure 4 One-way sensitivity analysis: the impact of STS screening uptake (\%) (Strategy A: maternal age with STS (age-STS) screening, Strategy B: STS plus NIPT screening, Strategy C: age-STS plus NIPT screening, Strategy D: maternal age with NIPT screening and Strategy E: universal NIPT screening). DS, Down syndrome; NIPT, non-invasive prenatal testing; STS, second-trimester triple screening.

Strategy D (figure 8). This means that, on that occasion, Strategy E could be the best choice for pregnant women to screen fetuses with Down syndrome.

\section{Probabilistic sensitivity analyses}

The cost-effectiveness planes for the 1000 Monte Carlo simulation (figures 9 and 10) show the results from PSA for the optimal strategy in the model analysis (Strategy D) and the strategy implemented in Fuyang City (Strategy E) vs the current strategy that is followed in China (Strategy C). Figure 9 shows a few spots $(34.3 \%)$ in the fourth

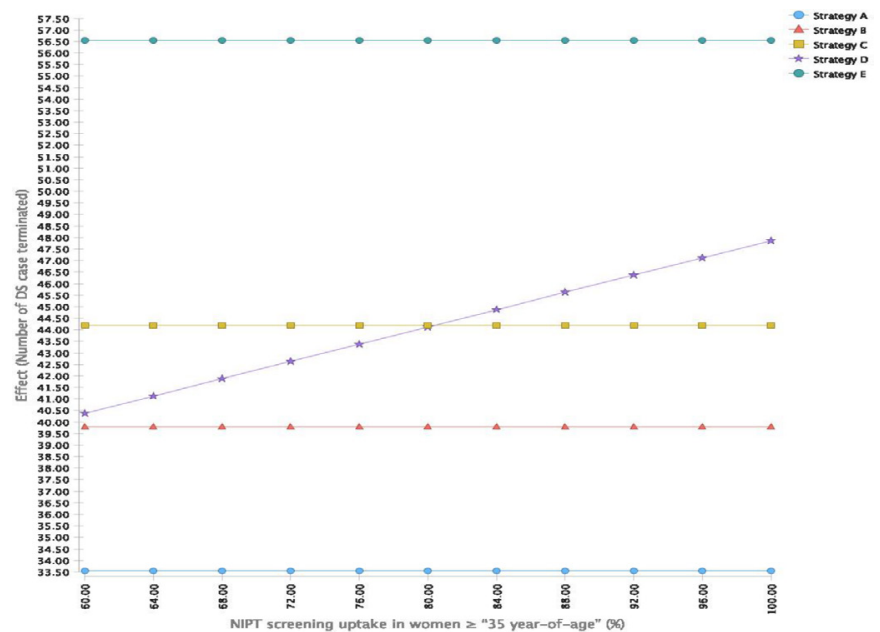

Figure 5 One-way sensitivity analysis: the impact of the NIPT screening uptake in women $\geq 35$ years of age (\%) (Strategy A: maternal age with STS (age-STS) screening, Strategy B: STS plus NIPT screening, Strategy C: age-STS plus NIPT screening, Strategy D: maternal age with NIPT screening, Strategy E: universal NIPT screening). DS, Down syndrome; NIPT, non-invasive prenatal testing; STS, secondtrimester triple screening.

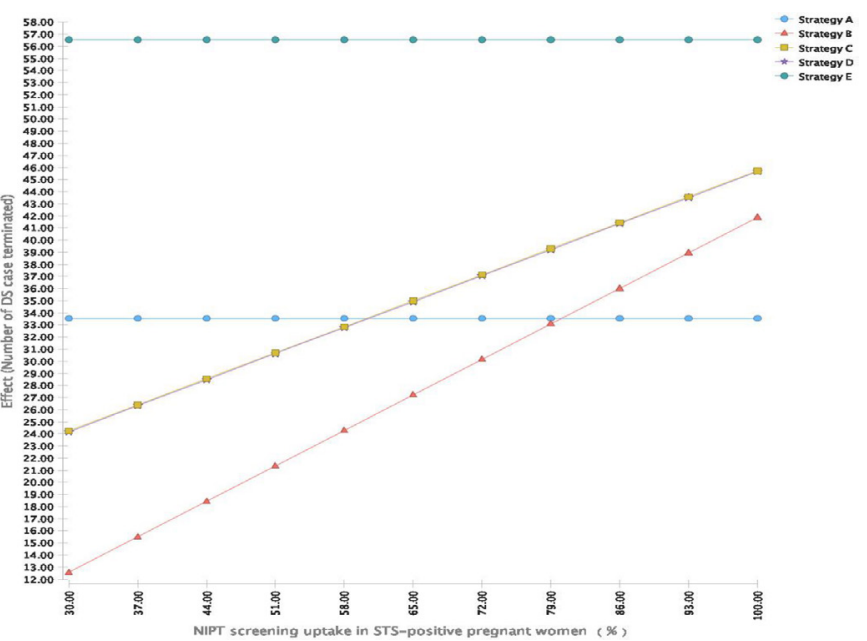

Figure 6 One-way sensitivity analysis: the impact of NIPT uptake in STS-positive pregnant women (\%) (Strategy A: maternal age with STS (age-STS) screening, Strategy B: STS plus NIPT screening, Strategy C: age-STS plus NIPT screening, Strategy D: maternal age with NIPT screening and Strategy E: universal NIPT screening). DS, Down syndrome; NIPT, non-invasive prenatal testing; STS, second-trimester triple screening.

quadrant, where Strategy D was more effective and less costly than Strategy C, and most spots (65.7\%) fell in the third quadrant, where Strategy D was less costly than Strategy C. Figure 10 shows that most spots (91.1\%) fell in the first quadrant, where Strategy E was more effective and costly than Strategy C.

The CEACs compare the probabilities of being costeffective for each strategy under different willingness to pay (WTP) per additional case of Down syndrome averted. Figure 11 shows that the most likely cost-effective strategy initially started with Strategy B and switched to Strategy

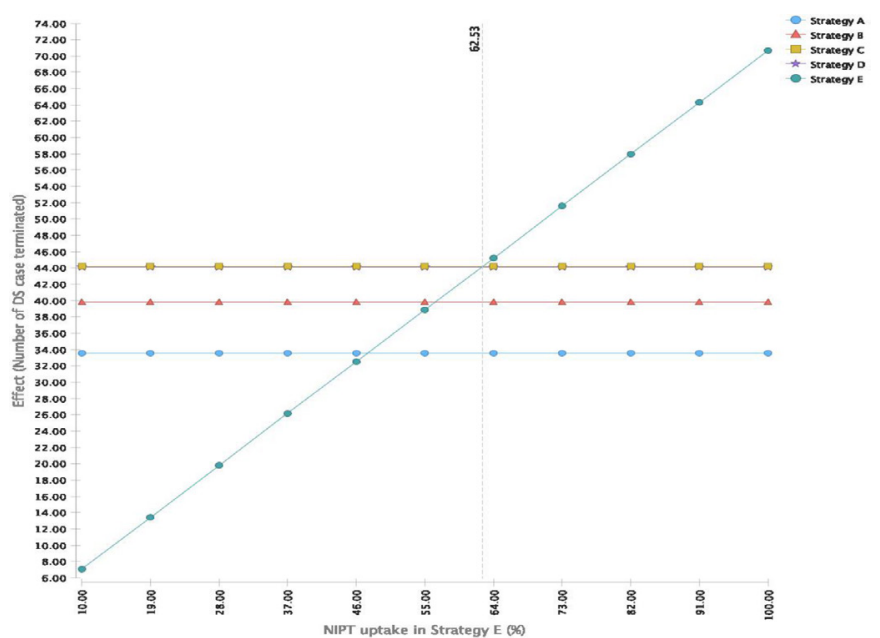

Figure 7 One-way sensitivity analysis: the impact of NIPT uptake in Strategy E (\%). (Strategy A: maternal age with STS (age-STS) screening, Strategy B: STS plus NIPT screening, Strategy C: age-STS plus NIPT screening, Strategy D: maternal age with NIPT screening and Strategy E: universal NIPT screening). DS, Down syndrome; NIPT, non-invasive prenatal testing; STS, second-trimester triple screening. 


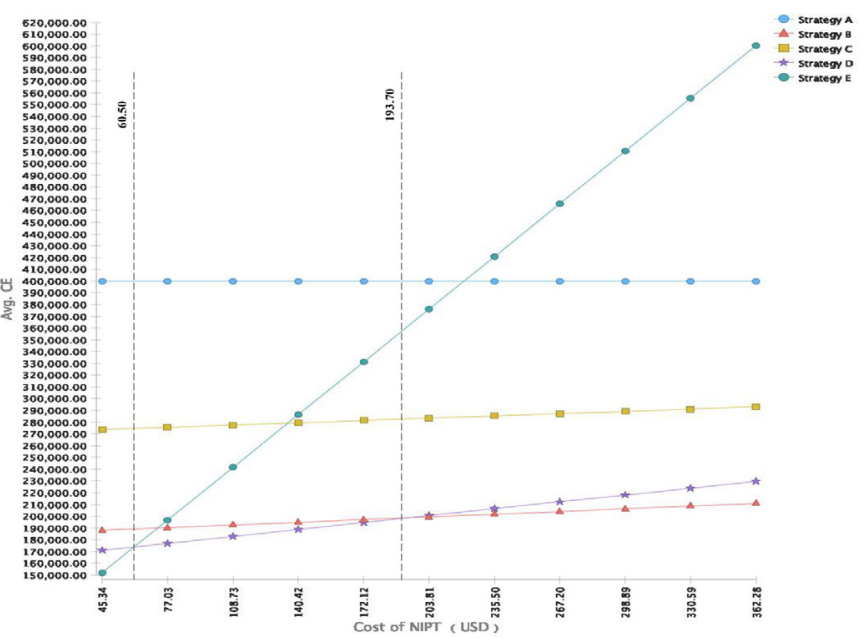

Figure 8 One-way sensitivity analysis: the impact of the cost of NIPT (Strategy A: maternal age with STS [age-STS] screening, Strategy B: STS plus NIPT screening, Strategy C: age-STS plus NIPT screening, Strategy D: maternal age with NIPT screening, and Strategy E: universal NIPT screening). CE, cost effectiveness; NIPT, non-invasive prenatal testing; STS, second-trimester triple screening.

D at thresholds of WTP starting at US $\$ 110$ 000.00. For thresholds of WTP per additional case of Down syndrome averted that exceeded US\$535 000.00, Strategy E had the highest probability of being cost-effective.

\section{DISCUSSION}

Introducing NIPT in prenatal screening for Down syndrome is more beneficial than conventional maternal serum screening strategy

This cost-effectiveness analysis from a societal perspective compared five different screening strategies that covered Down syndrome screening strategies in different

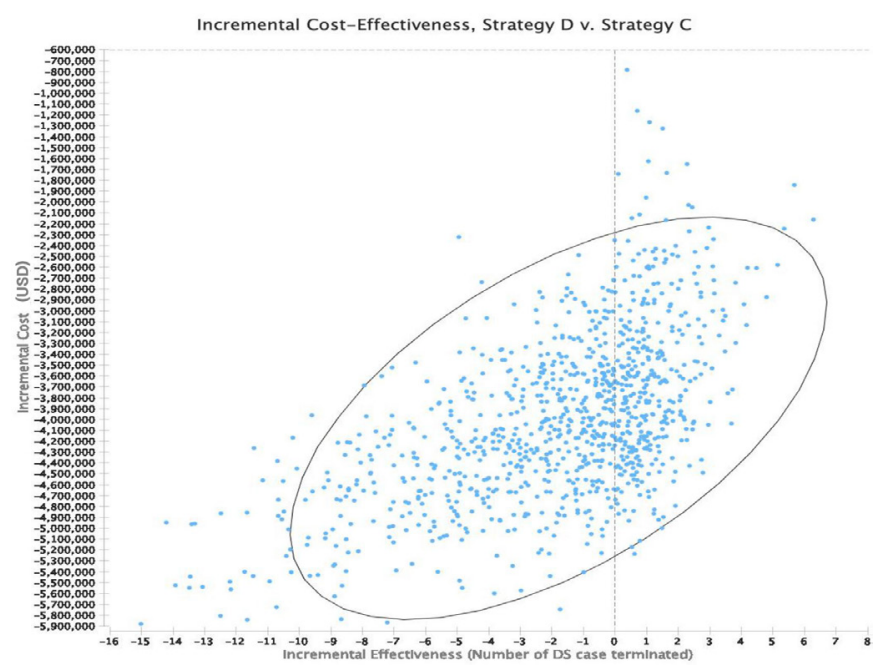

Figure 9 Incremental cost-effectiveness scatter plot (Strategy D vs Strategy C) (Strategy D: maternal age with NIPT screening, Strategy C: age-STS plus NIPT screening). DS, Down syndrome; NIPT, non-invasive prenatal screening; STS, second-trimester triple screening.

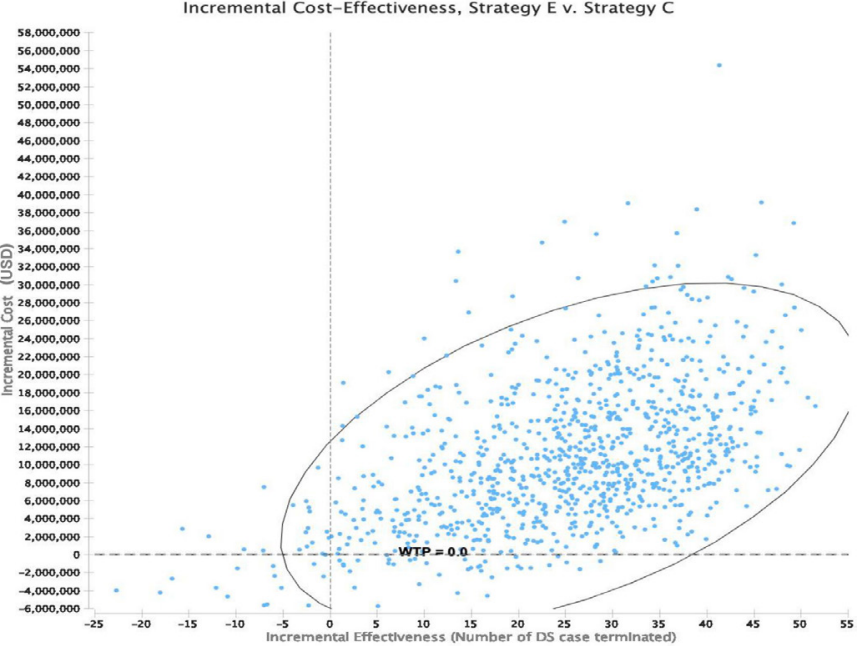

Figure 10 Incremental cost-effectiveness scatter plot (Strategy E vs Strategy C) (Strategy C: age-STS plus NIPT screening, Strategy E: universal NIPT screening). DS, Down syndrome; NIPT, non-invasive prenatal testing; STS, secondtrimester triple screening.

regions of China. Meanwhile, using context-specific realworld information on probability and cost parameters from the survey in Fuyang City allows an accurate evaluation in a policy-specific context. Our results demonstrate that, at the current NIPT screening uptake rate and the price of NIPT paid through a government-funded programme, introducing NIPT in prenatal screening strategies for Down syndrome was beneficial over the STS strategy. NIPT with a high DR and a low FPR in prenatal screening for Down syndrome identifies more Down syndrome cases, provides more efficient referral for invasive testing, avoids unnecessary PRL, decreases live-birth Down syndrome cases and saves costs per Down syndrome

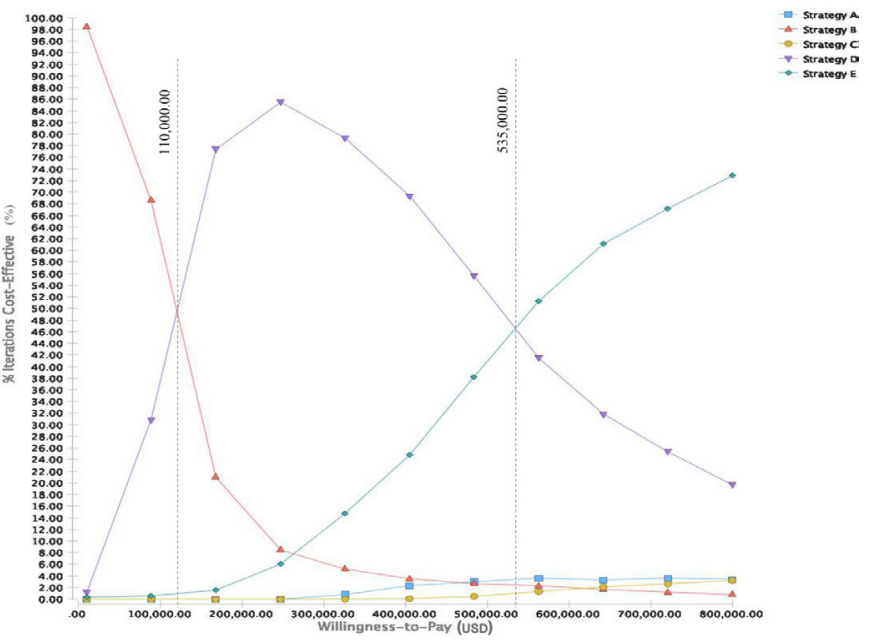

Figure 11 Cost-effectiveness acceptability curve: Five screening strategies (Strategy A: maternal age with STS (age-STS) screening, Strategy B: STS plus NIPT screening, Strategy C: age-STS plus NIPT screening, Strategy D: maternal age with NIPT screening and Strategy E: universal NIPT screening). NIPT, non-invasive prenatal testing; STS, second-trimester triple screening. 
case that is detected. In our study, Strategy D was the most optimal choice, instead of Strategy C (the currently referred screening strategy in China). Furthermore, universal NIPT screening (Strategy E) is the most effective option and shows several clinical benefits, although it is also the most expensive strategy compared with other alternative strategies. This result is consistent with some previous cost-effectiveness analysis studies in other countries, including the USA, Australia and some European countries. ${ }^{1922} 23$

Compared with the current screening strategy (Strategy C), maternal age with NIPT screening strategy (Strategy D) may be a better prenatal screening strategy for Down syndrome in China

The current referred screening strategy (Strategy C) directly provided invasive diagnostic tests to pregnant women with advanced maternal age ( $\geq 35$ years), whereas Strategy D offered NIPT first, followed by invasive diagnostic tests for NIPT-positive pregnant women with advanced maternal age. Thus, Strategy D can reduce unnecessary PRLs caused by invasive diagnostic tests. As mentioned in the results of base case analysis, Strategies $\mathrm{D}$ and $\mathrm{C}$ detected almost the same number of Down syndrome cases (44.12 and 44.20, respectively). However, Strategy D was associated with significantly less miscarriages following invasive procedures ( 0.14 vs 16.52$)$. Thus, these two strategies had similar effects, but Strategy D was safer for pregnant women. In clinical practice, the safety of the screening strategy is one of the most important factors. ${ }^{24}$ Furthermore, Strategy D has a lower CE ratio than Strategy C, which is the most recommended prenatal screening strategy for Down syndrome in China. However, given the safety and cost-saving concerns, Strategy D may be a better choice, especially for pregnant women with advanced maternal age who have a higher risk of Down syndrome fetuses. Furthermore, the CEACs indicate that, at a threshold below US $\$ 535000.00$, Strategy D was always more likely to be cost-effective when compared with Strategy C. This positive evidence might facilitate better resource allocation, considering the extremely limited WTP threshold information in China.

Reducing the price of NIPT, universal NIPT screening (Strategy E) could become a priority choice

In the context that NIPT was approved and funded by the government and freely offered to each eligible pregnant woman, Strategy E was not the optimal choice compared with the other strategies at the existing price. The sensitivity analysis indicated that the results were sensitive to the screening uptake rate and cost per NIPT. If the screening adherence of NIPT can be improved to $62.53 \%$, Strategy E would be able to detect the maximum number of Down syndrome cases. The context-specific real-world survey in Fuyang City showed that NIPT screening uptake substantially increased to $80 \%$. Furthermore, the sensitivity analysis showed that when the cost was less than US\$60.50, the CE ratio of Strategy E was the lowest compared with the other strategies. Fuyang City implemented Strategy E, and the cost of NIPT was
US $\$ 112.58$ for each pregnant woman, which is relatively low because of the local government's price negotiation. However, local governments might face fiscal pressure if they fully fund the programme in the long term, unless the unit cost of NIPT can be further reduced. Further regulation of the cost of NIPT might be achieved by the national health authority through negotiations with the private sector. Moreover, exploiting better payment methods, such as cost sharing by the government and the patient, might ensure the sustainability of the project.

Some limitations of the study must be considered when interpreting of results. First, the analysis was conducted based on a province in China, whereas the screening in actual clinical practice and costs can be quite different from those in other countries. Therefore, the results must be interpreted cautiously in the context of other settings. However, these findings may provide evidence for other developing countries with similar prenatal screening and diagnostic policies. Second, our study focused only on fetal trisomy 21. Including trisomy 18 and 13 in the model may confer greater clinical benefits of NIPT due to the better detection and less reliance on unnecessary invasive procedures for these aneuploidies. However, such an inclusion will greatly increase the complexity of the model and affect the reliability of the model due to scarce clinical data for aneuploidies. Third, we assumed that the uptake of invasive diagnostic testing after a positive NIPT result was $100 \%$, but it may be lower in clinical practice. For social or religious reasons or out of the fear of miscarriage, some pregnant women do not accept any invasive procedure even when genetic counsellors recommend further testing. Even though assumptions have been made, the decision-analytical modelling can still be used to guide better allocation of resources.

\section{CONCLUSION}

In summary, our economic analysis from a societal perspective of China's pregnant population suggests that the introduction of NIPT as a screening strategy for Down syndrome would be more cost-effective than the use of STS. Given the safety and cost-saving benefits, factoring in the maternal age with the NIPT screening strategy may be a better choice than the currently referred screening strategy in China and should be recommended to pregnant women, especially those who are older than 35 years. The universal NIPT strategy can identify a higher number of Down syndrome cases, reduce unnecessary invasive procedures and incur the highest cost. The NIPT screening strategy is still non-optimal at the current price in the government-funded projects; however, our results indicate that lowering the price of NIPT is an effective measure to promote the acceptance of a universal NIPT strategy.

Contributors $\mathrm{JH}$ conceived the idea of this research and contributed to the writing of this manuscript. WS developed and designed the economic model, conducted a cost-effectiveness analysis, undertook data collection and wrote this manuscript. YW provided clinical guidance and undertook the data collection for the economic 
model. JC contributed to the writing of the manuscript. YD provided guidance for devising the study methodology. All authors approved the final version of the manuscript.

Funding This study was funded by the national Key Research and Development Program of China (Grant ID: 2018YFC1002203).

Competing interests None declared.

Patient consent for publication Not required.

Ethics approval The study was approved by the Medical Research Ethics Committee, School of Public Health, Fudan University, 07/20/2018 (IRB00002408 \& FWA00002399).

Provenance and peer review Not commissioned; externally peer reviewed.

Data availability statement The data that support the findings of this study are available from the corresponding author upon reasonable request.

Supplemental material This content has been supplied by the author(s). It has not been vetted by BMJ Publishing Group Limited (BMJ) and may not have been peer-reviewed. Any opinions or recommendations discussed are solely those of the author(s) and are not endorsed by BMJ. BMJ disclaims all liability and responsibility arising from any reliance placed on the content. Where the content includes any translated material, BMJ does not warrant the accuracy and reliability of the translations (including but not limited to local regulations, clinical guidelines, terminology, drug names and drug dosages), and is not responsible for any error and/or omissions arising from translation and adaptation or otherwise.

Open access This is an open access article distributed in accordance with the Creative Commons Attribution Non Commercial (CC BY-NC 4.0) license, which permits others to distribute, remix, adapt, build upon this work non-commercially, and license their derivative works on different terms, provided the original work is properly cited, appropriate credit is given, any changes made indicated, and the use is non-commercial. See: http://creativecommons.org/licenses/by-nc/4.0/.

ORCID iD

Jiayan Huang http://orcid.org/0000-0003-4166-6119

\section{REFERENCES}

1 Kazemi M, Salehi M, Kheirollahi M. Down syndrome: current status, challenges and future perspectives. Int $\mathrm{J} \mathrm{Mol} \mathrm{Cell} \mathrm{Med}$ 2016;5:125-33.

2 Audibert F, De Bie I, Johnson J-A, et al. No. 348-Joint SOGC-CCMG guideline: update on prenatal screening for fetal aneuploidy, fetal anomalies, and adverse pregnancy outcomes. J Obstet Gynaecol Can 2017:39:805-17.

3 Mackie FL, Hemming K, Allen S, et al. The accuracy of cellfree fetal DNA-based non-invasive prenatal testing in singleton pregnancies: a systematic review and bivariate meta-analysis. BJOG 2017;124:32-46.

4 Jin J, Yang J, Chen Y, et al. Systematic review and meta-analysis of non-invasive prenatal DNA testing for trisomy 21: implications for implementation in China. Prenat Diagn 2017;37:864-73.

5 Tu S, Rosenthal M, Wang D, et al. Performance of prenatal screening using maternal serum and ultrasound markers for Down syndrome in Chinese women: a systematic review and meta-analysis. BJOG 2016;123 Suppl 3:12-22.

6 Salomon LJ, Sotiriadis A, Wulff CB, et al. Risk of miscarriage following amniocentesis or chorionic villus sampling: systematic review of literature and updated meta-analysis. Ultrasound Obstet Gynecol 2019;54:442-51.

7 Gregg AR, Skotko BG, Benkendorf JL, et al. Noninvasive prenatal screening for fetal aneuploidy, 2016 update: a position statement of the American College of medical genetics and genomics. Genet Med 2016;18:1056-65.

8 UK National Screening Committee. UK NSC non-invasive prenatal testing (NIPT) recommendation, 2016. Available: https:// legacyscreening.phe.org.uk/fetalanomalies [Accessed 8 May 2020]

9 Dashe JS. Aneuploidy screening in pregnancy. Obstet Gynecol 2016;128:181-94.

10 National Health Commission of the People's Republic of China. Notice on orderly carrying out prenatal screening and diagnosis of fetal free DNA in maternal peripheral blood. Available: http:// www.nhc.gov.cn/fys/s3581/201611/0e6fe5bac1664ebda8bc28ad 0ed68389.shtml [Accessed 8 May 2020].

11 National Health Commission of the People's Republic of China. Notice on strengthening supervision and management of prenatal screening and diagnosis for fetal free DNA in maternal peripheral blood. Available: http://www.nhc.gov.cn/fys/s3589/201911/da796e1f 1e7745e7b52198474d9a6cc5.shtml [Accessed 8 May 2020].

12 Chinese Health Quality Management. Measures for the implementation of the maternal and child health care law of the People's Republic of China; 2002: 5-8.

13 Bunnik EM, Kater-Kuipers A, Galjaard R-JH, et al. Why NIPT should be publicly funded. J Med Ethics 2020;46:783-4.

14 Alberry MS, Aziz E, Ahmed SR, et al. Non invasive prenatal testing (NIPT) for common aneuploidies and beyond. Eur J Obstet Gynecol Reprod Biol 2021;258:424-9.

15 Benoy ME, Iruretagoyena JI, Birkeland LE, et al. The impact of insurance on equitable access to non-invasive prenatal screening (NIPT): private insurance may not pay. J Community Genet 2021;12:185-97.

16 Samura O. Update on noninvasive prenatal testing: a review based on current worldwide research. J Obstet Gynaecol Res 2020;46:1246-54.

17 Zhu W, Ling X, Shang W, et al. The knowledge, attitude, practices, and satisfaction of non-invasive prenatal testing among Chinese pregnant women under different payment schemes: a comparative study. Int J Environ Res Public Health 2020;17:7187.

18 Walker BS, Nelson RE, Jackson BR, et al. A cost-effectiveness analysis of first trimester non-invasive prenatal screening for fetal trisomies in the United States. PLoS One 2015;10:e0131402.

19 García-Pérez L, Linertová R, Álvarez-de-la-Rosa M, et al. CostEffectiveness of cell-free DNA in maternal blood testing for prenatal detection of trisomy 21, 18 and 13: a systematic review. Eur $J$ Health Econ 2018;19:979-91.

20 Hulstaert F, Neyt M, Gyselaers W. The non-invasive prenatal test (NIPT) for trisomy 21-health economic aspects. Belgian Health Care Knowledge Centre (KCE): Health Technology Assessment (HTA) Brussels, 2014.

21 Badeau M, Lindsay C, Blais J, et al. Genomics-Based non-invasive prenatal testing for detection of fetal chromosomal aneuploidy in pregnant women. Cochrane Database Syst Rev 2017;11:CD011767.

22 Morris S, Karlsen S, Chung N, et al. Model-Based analysis of costs and outcomes of non-invasive prenatal testing for Down's syndrome using cell free fetal DNA in the UK National health service. PLoS One 2014;9:e93559.

23 Evans MI, Sonek JD, Hallahan TW, et al. Cell-Free fetal DNA screening in the USA: a cost analysis of screening strategies. Ultrasound Obstet Gynecol 2015;45:74-83.

24 Bowman-Smart H, Savulescu J, Mand C, et al. 'Small cost to pay for peace of mind': women's experiences with non-invasive prenatal testing. Aust N Z J Obstet Gynaecol 2019;59:649-55.

25 Chen YM, Lu S, Zhang W. Analysis of incidence and epidemiological characteristics of Down syndrome in Hangzhou. Chinese Journal of Birth Health \& Heredity 2019;27:688-91. Chinese.

$26 \mathrm{HY}$ X, Liu KB, Qi Q. Analysis of screening of Down syndrome in Beijing from 2010 to 2014. Chinese Journal of Birth Health \& Heredity 2016;24:48-9. Chinese.

27 National Maternal and Child Health Office, the Department of Maternal and Child Health Services,, National Health Commission. The National maternal and child health surveillance and annual report Newsletter No. 5 in 2018. (Chinese).

28 Li C, Shi L, Huang J, et al. Factors associated with utilization of maternal serum screening for Down syndrome in mainland China: a cross-sectional study. BMC Health Serv Res 2015;16:8.

29 Yang L, Zhao L, Jiang J. [Serum marker screening during the second trimester for prenatal diagnosis and predicting pregnancy outcome]. Nan Fang Yi Ke Da Xue Xue Bao 2015;35:1059-62.

30 Deng B, Qiu MZ, Qiu ZH. Value of serum triple screening combined with non-invasive prenatal testing in the diagnosis of Down's syndrome. Journal of Guangdong MedicalUniversity 2018;36:404-6.

31 Yao JY, Cui L, Yue S. Contingent prenatal screening in second trimester: using a simple two serum marker and non-invasive prenatal testing. The Journal of Medical Theory and Practice 2018;31:3330-2.

32 Liao C, Wei J, Li Q, et al. Efficacy and safety of cordocentesis for prenatal diagnosis. Int J Gynaecol Obstet 2006;93:13-17.

33 Mansfield C, Hopfer S, Marteau TM. Termination rates after prenatal diagnosis of Down syndrome, spina bifida, anencephaly, and Turner and Klinefelter syndromes: a systematic literature review. Prenat Diagn 1999;19:808-12.

34 Savva GM, Morris JK, Mutton DE, et al. Maternal age-specific fetal loss rates in Down syndrome pregnancies. Prenat Diagn 2006;26:499-504.

35 Liu B, Gao E. Risk factors for spontaneous abortion of Chinese married women at reproductive age. China Public Health 2002;18:890-2. 